VERNIER Bernard, Tu veux qu'on sorte ensemble? La transformation des formes de flirt dans six villages musulmans de Grèce

Toulouse, Presses Universitaires du Mirail, «Les anthropologiques », 2013, 414 p., ISBN : 978-2-8107-0253-4.

Katerina Seraïdari

\title{
CpenEdition
} Journals

Édition électronique

URL : https://journals.openedition.org/ceb/9076

DOI : 10.4000/ceb.9076

ISSN : 2261-4184

Éditeur

INALCO

Édition imprimée

ISBN : 978-2-85831-229-0

ISSN : 0290-7402

Référence électronique

Katerina Seraïdari, " vernier Bernard, Tu veux qu'on sorte ensemble? La transformation des formes de flirt dans six villages musulmans de Grèce », Cahiers balkaniques [En ligne], 43 | 2015, mis en ligne le 07 juin 2016, consulté le 06 juillet 2021. URL : http://journals.openedition.org/ceb/9076 ; DOI : https://doi.org/ 10.4000/ceb.9076

Ce document a été généré automatiquement le 6 juillet 2021.

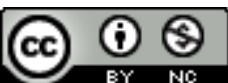

Cahiers balkaniques est mis à disposition selon les termes de la Licence Creative Commons Attribution - Pas d'Utilisation Commerciale 4.0 International. 


\section{VERNIER Bernard, Tu veux qu'on sorte ensemble? La transformation des formes de flirt dans six villages musulmans de Grèce}

Toulouse, Presses Universitaires du Mirail, «Les anthropologiques », 2013, 414 p., ISBN : 978-2-8107-0253-4.

\section{Katerina Seraïdari}

1 Ce livre analyse les formes publiques de flirt et leur évolution dans des villages musulmans situés au Nord de la Grèce, dans la région de Xanthi, près de la frontière bulgare. L'auteur définit ainsi son objet d'étude : «la naissance et la transformation d'une institution de rencontre entre les jeunes ", qui a lieu en plein air, "de façon quotidienne, en dehors de tout travail et indépendamment de toute fête religieuse » (p.380). Vernier avait effectué des enquêtes ethnographiques dans cette région pendant deux mois en 1966-1967, avant d'y revenir à l'été 1997 pour étudier la transformation des rapports entre les deux sexes. Les données ethnographiques qu'il nous livre ici sont les résultats de cette enquête, qui a été poursuivie pendant l'été 2008 et l'été 2010 , et qui a duré, en tout, trois mois (p. 376, note 64). Son observation participante "répétée à intervalles assez courts » lui a permis, comme il le souligne, d'entrer « dans le moteur de l'institution », celle-ci étant modifiée en permanence par «petites couches transgressives» (p. 381).

2 Au centre de l'analyse se trouvent donc les mécanismes de fonctionnement du marché matrimonial, ainsi que le processus de libéralisation des mœurs et d'occidentalisation des valeurs de cette société musulmane. Les tenues vestimentaires des jeunes sont discutées en détail, ainsi que d'autres facteurs de changement: la diffusion des motocyclettes (grâce à l'argent du travail salarié en Grèce ou à l'étranger), le rôle de l'émigration, l'unification du marché matrimonial régional grâce à cette mobilité accrue, les rapports de force entre différents types d'élites (religieuses ou non), les effets de la scolarisation (surtout celle des filles dans le secondaire), l'influence des 
séries télévisées, les types de commerces qui facilitent le rassemblement des jeunes et le rôle de la musique (les paroles des chansons remplaçant les déclarations d'amour), l'omniprésence des téléphones portables, les graffitis qui rendent publiques les affaires amoureuses. Vernier montre comment les graffitis qui associent « des initiales ou des prénoms par le signe plus» conduisent à une perte de romantisme et à "un affaiblissement de l'idéalisation des relations amoureuses" (p. 258); et comment le téléphone portable supprime la dépendance vis-à-vis d'intermédiaires, tout en permettant de s'émanciper de la pression de l'opinion publique villageoise (p. 233 et p. 299).

3 Dans ce cadre, la tenue vestimentaire parvient à définir l'individu dans son intimité personnelle : une femme qui porte le foulard est «fermée » (klisti en grec), tandis que celle qui ne le porte pas est « ouverte » (anihti). Vernier analyse les différents types de foulards (p. 130-131) : de celui qui est «allusif » et laisse voir une partie des cheveux, à celui qui a la forme d'un turban, dont la mode vient de Turquie et que certains musulmans laïcs de Xanthi qualifient d'« intégriste» (p. 135 et p. 369). Selon lui, le fait d'interdire le foulard ou de l'imposer alimente un même "fétichisme du foulard" (p. 389). Pour sortir de ce qu'il considère être un faux dilemme, il introduit la notion de " loi synchronique de compensation " : « toute modernisation importante des pratiques dans un domaine (vestimentaire, interactions entre les sexes, etc.) se paie très souvent du maintien d'un comportement traditionnel dans le même domaine ou dans un autre domaine" (p.387). Il considère, d'une part que le foulard "autrefois symbole d'aliénation devient un instrument de libération» (p. 239), et d'autre part, qu'il est associé à «une morale de l'appartenance et non d'intention» (p. 366). En effet, il constitue un marqueur à l'intérieur de la société musulmane, mais aussi en ce qui concerne la distinction entre chrétiens et musulmans : quand une fille enlève son foulard, elle est accusée d'avoir changé de croyance et d'être devenue chrétienne (p. 347, p. 363 et p. 366). De même, une fille avec un large décolleté se plaint d'être qualifiée de «chrétienne » (p. 386). Dans un autre récit, les parents d'une jeune fille qui a subi une opération n'envisagent même pas l'éventualité d'une transfusion, car ils "auraient préféré qu'elle meure plutôt que de lui mettre du sang de chrétien » (p. 363-364).

4 L'aspect comparatif et l'étude des pratiques de flirt dans plusieurs villages permettent à l'auteur de distinguer entre ceux qui sont plus traditionnels et ceux qui le sont moins. Dans un village, dont les habitants, qui sont très religieux, n'ont commencé à émigrer massivement que vers 1990, toutes les filles portent le foulard à partir de 10 ou 12 ans, mais il n'est pas rare de le voir porté par des filles de 5 ou 6 ans (p. 138). En fait, les jeunes que Vernier met au centre de son étude ne sont pas très bien définis en tant que catégorie. Par exemple, dans ce village, l'auteur constate à un moment donné la présence de deux groupes de filles, séparés en classes d'âge: les plus grandes (de 12 à 25 ans) et les plus petites (de 4 ou 5 ans à 12 ans environ). Dans un autre village qui a connu une émigration précoce, il parle d'un rassemblement de trente à quarante garçons qui ont aux environs de 25 ans, et d'autant de filles qui ont entre 7 et 8 ans (p. 198). Ces données laissent supposer que les filles entrent dans ce type d'interactions très tôt, entre 4 et 7 ans, et se retirent également assez tôt, vers l'âge de 25 ans (p. 189). La question qui se pose est de savoir si on peut parler de flirt dans le cas de filles si jeunes. Vernier considère que la présence de très petits enfants donne « à la rencontre un caractère innocent » (p. 110 et p. 210). Ceci étant dit, pourquoi ces rapports qui se nouent avec l'autre sexe depuis l'enfance jusqu'au mariage devraient-ils s'inscrire dans 
un registre érotique de séduction? Pourquoi ne pas simplement voir des occasions de s'amuser, de faire connaissance et de nouer des amitiés avec le sexe opposé ? Comme l'auteur le dit, on est parfois « dans une ambiance de cour de récréation où fusent rires et plaisanteries » (p. 219), d'autant plus que la scolarisation «dans des établissements mixtes qui encourage la camaraderie entre les sexes» (p. 232) facilite la manifestation de tels comportements.

5 Ces relations révèlent plus largement l'émergence d'une culture des jeunes, qui a ses propres codes et qui se manifeste dans certains espaces. En effet, Vernier montre comment les changements qu'il étudie "ont transformé les rapports entre les générations [...] et entre les sexes", en garantissant une plus grande égalité entre ces derniers (p. 105). Dans ce cadre, les petites filles, à qui «le rôle d'avant-garde de la mode villageoise " est attribué, sont celles qui s'habillent de la manière la plus moderne : elles annoncent la façon dont les grandes s'habilleront dans les années à venir (p. 214). Mais des filles de 4 à 8 ans ne font pas leurs achats vestimentaires seules; elles ne s'habillent pas non plus sans être soumises au contrôle de leur mère ou de leur sœur aînée. Ne sont-ce pas les mères qui habillent ces petites filles qui sont à l'avantgarde de la mode ? En général, l'absence de définition de la catégorie des jeunes tend à les assimiler aux célibataires, les personnes mariées étant automatiquement catégorisées comme adultes. Cependant, le mariage, même s'il constitue une rupture dans un parcours biographique, ne doit pas cacher la différence entre ceux qui sont mariés et ont 25 ans et ceux qui en ont 60 . Pourquoi une femme mariée de 25 ans qui habille sa fille de 4 ans de manière moderne serait-elle plus éloignée de la culture des jeunes que sa propre petite fille? Pourquoi, quand un homme marié promène sa femme et ses enfants en moto, exhibe-t-il « une forme progressiste de paternité et de relation maritale» (p. 223) et ne reproduit-il pas, tout simplement, ce qu'il faisait, quelques années auparavant, lors de ses fiançailles ? Vernier, qui constate " une atténuation de la barrière hiérarchique générationnelle » (p. 230), nous montre précisément comment la structure des rapports entre générations change, à l'instar de ces filles qui « boivent de la liqueur avec leur mère et se mettent à rire tellement qu'elles cassent leur verre. Leurs mères se comportent comme si elles étaient leurs sœurs » (p. 229).

6 Avec ses enquêtes ethnographiques denses et ses analyses subtiles, Vernier capte une société en pleine mutation. Ce travail original montre comment des jeunes musulmans de nationalité grecque (souvent appelés « Pomaques ", terme qui n'apparaît pas dans le sous-titre car rejeté par les interlocuteurs de Vernier) gagnent en autonomie sous l'effet de la mondialisation.

\section{AUTEUR}

\section{KATERINA SERAÏDARI}

Centre d'Anthropologie sociale, LISST, Toulouse 